\title{
Mathematical Models of Receptivity of a Robot and a Human to Education
}

\author{
Oleg G. Pensky, Vladimir O. Michailov, Kirill V. Chernikov \\ Perm State University, Perm, Russia \\ Email: ogpensky@mail.ru
}

Received 20 May 2014; revised 23 June 2014; accepted 2 July 2014

Copyright (C) 2014 by authors and Scientific Research Publishing Inc. This work is licensed under the Creative Commons Attribution International License (CC BY). http://creativecommons.org/licenses/by/4.0/

\section{(c) (i) Open Access}

\begin{abstract}
The paper gives general definitions of the mathematical theory of emotional robots able to forget older information. A formalized concept of relative receptivity of the robot to education is introduced. An algorithm of a voice training program for public speakers described in the paper is based on the theory of emotional robots. Also the paper presents a method of estimation of a coefficient of human emotional memory and estimation of a relative receptivity of a robot and a human to education; the method is based on application of the voice training program.
\end{abstract}

\section{Keywords}

Robot, Robot's Education, Receptivity to Education, Memory, Emotions of Robots

\section{Introduction}

According to forecasts, by 2018 the world market of humanoid robots has to make 25.5 billion dollars. For the process of building such robots it is important to develop a mathematical tool and the software simulating an "emotional" sphere of functioning of human-like robots.

Suppose the robot experiences emotions.

\section{Methods}

Assume the robot's emotion has a form of a certain integrated function $M_{i}(\tau)$ where $\tau$ is the current time of emotional effect, $\tau \in[0, T], T$ is the step i.e. the time step which is the duration of emotion, $i$ is the serial number of an emotion experienced by the robot.

Let us give several definitions introduced in [1].

Definition 1. The robot's elementary education $r(\tau)$ is a function of the following form: 


$$
r_{i}(\tau)=\int_{0}^{\tau} M_{i}(\xi) \mathrm{d} \xi
$$

Assume the robot experiences emotions continuously.

Definition 2. The robot's education $R_{i}(t)$ is a function of the following form:

$$
R_{i}(t)=r_{i}(\tau)+\theta_{i}(t) R_{i}\left(t_{i}\right)
$$

where $t$ is the current time of the robot's education, $t>t_{i-1}, 0 \leq \theta_{i}(t) \leq 1$. The current time satisfies the relation $t=\tau+t_{i-1}$, where $\tau$ is the current time of effect of the current emotion from the beginning of its manifestation, $t_{i-1}$ is the general time of effect of all the previous emotions, $R_{i}\left(t_{i}\right)$ is the education obtained by the robot during the time $t_{i}$.

The coefficients $\theta_{i}(t)$ are coefficients of the robot's memory. It should be noted that the robot's memory coefficients determine that part of the former (previous) education of the robot remembered by the latter.

According to (1) we can write down a formula defining the robot's education at the end of the $i$-th step [2]:

$$
R_{i}\left(t_{i}\right)=r_{i}\left(t_{i}\right)+\theta_{i}\left(t_{i}\right) R_{i-1}\left(t_{i-1}\right) .
$$

Equation (1) can be written down in the following form:

$$
R_{i}(t)=r_{i}(\tau)+\theta_{i}(t) r_{i-1}+\theta_{i}(t) \theta_{i-1}(t) r_{i-2}+\cdots+\theta_{i}(t) \theta_{i-1}(t) \theta_{i-2}(t) \cdots \theta_{1}(t) r_{0} .
$$

Definition 3. Emotions initiating equal elementary educations at the end of the time step are called tantamount emotions.

Definition 4. A uniformly forgetful robot is a forgetful robot whose memory coefficients corresponding to the end time points of each emotion are constant and equal.

Assume that for tantamount emotions of the uniformly forgetful robot at the end of each step the relations $r_{i}\left(t_{i}\right)=q=$ const, $\theta_{i}\left(t_{i}\right)=\theta, i=1, \bar{\infty}$ are true.

Then, according to the formula of the sum $i$ of terms of geometric series, Relation (3) implies

$$
R_{i}\left(t_{i}\right)=q \frac{1-\theta^{i}}{1-\theta} .
$$

So, the formula

$$
\lim _{i \rightarrow \infty} q \frac{1-\theta^{i}}{1-\theta}=\frac{q}{1-\theta}
$$

is obviously true.

This limiting value is the robot's limiting education.

Obviously (3) - (5) are true only when the robot experiences emotions continuously: one after another. But the robot may have a break in experiencing emotions. In this case the robot forgets its last education. The following definition is introduced to describe this process.

Definition 5. A dummy step is a time interval during which the robot's education decreases by $\theta$ times.

A real educational process of the robot can obviously be approximated by the education process of the uniformly forgetful robot with tantamount emotions.

Let us have a look at an example.

Assume the values $R_{1}\left(t_{1}\right), R_{2}\left(t_{2}\right), \cdots, R_{n}\left(t_{n}\right)$ of the robot's education are defined at the end of each step and dummy step, and the robot's memory coefficient $\theta$ is also defined.

To estimate the educational process parameter $q$ of the uniformly forgetful robot with tantamount emotions it is enough to solve the following optimizing problem:

solve for

$$
\min _{q} \sum_{i=1}^{n}\left(R_{i}\left(t_{i}\right)-q-\theta R_{i-1}\left(t_{i-1}\right)\right)^{2}
$$

where $R_{0}\left(t_{0}\right)=R_{0}(0)=0$.

Applying methods of definition of extremum for single-variable functions we obtain the equality 


$$
q=\frac{\sum_{i=1}^{n} R_{i}\left(t_{i}\right)-\theta \sum_{i=1}^{n} R_{i-1}\left(t_{i-1}\right)}{n}
$$

which is the solution of Problem (6).

For alternating steps in a series "steps-dummy steps-steps" the formula of education of the uniformly forgetful robot with tantamount emotions $R_{i+j+k}\left(t_{i+j+k}\right)$ takes the form

$$
R_{i+j+k}\left(t_{i+j+k}\right) \approx q \frac{1-\theta^{k}}{1-\theta}+\theta^{j+k} q \frac{1-\theta^{i}}{1-\theta}
$$

where $i$ is the number of steps in the first series, $j$ is the number of dummy steps in the second series, $k$ is the number of the steps in the third series.

[3] is the first to introduce models of receptivity to the education $\varepsilon$ and relative receptivity to the robot's education $\alpha$.

If the condition $q>0$ is satisfied, then according to [3] receptivity to the education $\varepsilon$ of the uniformly forgetful robot with tantamount emotions satisfies the relation

$$
\varepsilon=\frac{q}{1-\theta}-R_{L}\left(t_{L}\right)
$$

where $q$ is the elementary education of robots with tantamount emotions, $q>0, \theta$ is the robot's memory coefficient, $R_{L}\left(t_{L}\right)$ is the robot's education at which the robot memorizes its last education which is defined by proximity to the limiting education.

According to [3] the relative receptivity to education can be written down in a form of the following equality:

$$
\alpha \approx \frac{\varepsilon}{\frac{q}{1-\theta}}=\frac{\varepsilon(1-\theta)}{q}
$$

It is easy to see that the relative receptivity $\alpha$ to education is a dimensionless quantity, $\alpha \in(0,1]$, and the less $\alpha$ is, the worse the robot's receptivity to education.

Suppose on the third round of the series of steps and dummy steps (second series of steps) the robot memorized the formerly received education.

Using (7) and (8) we obtain

$$
\varepsilon \approx \frac{q}{1-\theta}-q \frac{1-\theta^{k}}{1-\theta}-\theta^{j+k} q \frac{1-\theta^{i}}{1-\theta}
$$

Using (9) for estimation of the relative receptivity $\alpha$ to education taking into account (10) we obtain

$$
\alpha \approx \theta^{k}\left(1-\theta^{j}+\theta^{i+j}\right)
$$

Analyzing (11) we can conclude that $\theta^{j}$ and $\theta^{i+j}$ can be neglected at large values of $i$ and $j$, and the relative receptivity can be estimated as $\alpha \approx \theta^{k}$.

\section{Results}

Below we describe practical application of the obtained relations.

Let us dwell on the definition of memory coefficients of the human whose analog is the emotional robot. For this purpose we use the well-known software system Vibraimage-7 developed by ELSYS enterprises (St. Petersburg, Russia) [4]. Vibraimage-7 is a software system for analyzing the psychophysiological and emotional condition of a person. On the basis of microvibrations of the human's head read by the webcam connected to the computer this software system is able to define his or her emotional condition expressed by a value with a range from 0 to 100 .

For measuring memory coefficients, the examinee is placed into the isolated room with the webcam. The computer with the program system is installed in the room next door. The examinee is placed opposite to the webcam. During the experiment this person is supposed to be relaxed and not to think about anything. The rest of the instructions are also very simple- the examinee is to look at the webcam for about 2 minutes while the 
program is operating and until the operator tells him or her that the experiment is over. After the examinee confirms that he or she is ready the supervisor of the experiment gives a command to start the experiment and goes out of the room with the webcam to activate Vibraimage-7. Thus, the examinee spends two minutes in the isolated room without external irritants while the program system is working. The experiment takes 2 minutes, and the data of the examinee's emotional condition is read at one-minute interval.

When the program system cycle is done, the supervisor comes into the room to notify the examinee that the experiment is over. So, in the course of the experiment we can obtain two readings of the examinee's education values which reflect the emotional condition of the examinee varying with the course of time.

Assume that the equivalent of the examinee's emotional condition measured by means of Vibraimage-7 is the robot's education. Then, during the experiment we can obtain two values of education: $R_{1}\left(t_{1}\right), R_{2}\left(t_{2}\right)$. Considering that the examinee was not impacted during the procedure, based on the first two values of $R_{1}\left(t_{1}\right), R_{2}\left(t_{2}\right)$ and education model (2) with $r_{i}(\tau) \equiv 0$ we can find the memory coefficient

$$
R_{2}\left(t_{2}\right)=\theta R_{1}\left(t_{1}\right), \Rightarrow \theta=\frac{R_{2}\left(t_{2}\right)}{R_{1}\left(t_{1}\right)}
$$

Suppose we need to model the emotional behavior of the robot interacting with the person who produces an effect on the robot by a signal injection, for example, by means of a microphone built in the robot. Suppose the emotional stimulus for the robot is the volume of sound [5]. Thus, it is necessary to define the dependence between the robot's emotions arising in the course of its interaction with the examinee (person) and the volume of the sound signal generated by that person to affect the robot.

To define the dependence between the human's emotions and the sound volume, we developed the computer program describing the following situation: "only one robot and one human are involved in the interaction. The robot has to respond emotionally to the sound impact (audio signal) generated by the human”.

In [6] we can find the description of the SoundBot program [7] simulating the mimic emotional response of the robot to audio stimuli. According to the description of the program functionality, it can be used by public speakers for voice training.

In this program, the speaker is listened (and estimated) by the robot with a non-absolute memory [1] which is capable for responding emotionally to the speaker's performance similar to the emotional reaction of a human listener.

Thus, the voice training technique is reduced to the following steps:

1) Set the upper and lower thresholds (bounds) of the robot's positive emotion defining the voice volume range within which the voice is to be trained.

2) Start the process of training of the speaker. In the course of this process the robot receives audio stimuli until only $i$ positive emotions are generated going sequentially one after another.

3) Human-robot interaction is interrupted for a period of $j$ dummy steps.

4) The speaker's voice is tested until the robot responses with a first positive emotion; this period takes $k$ steps.

On the basis of the methods described above we performed a series of experiments on voice training of several speakers with predetermined memory coefficients. The results of these experiments and the corresponding values of relative receptivity $\alpha$ of robots to education are presented in Table 1.

Table 1. Memory coefficients and relative receptivity to education.

\begin{tabular}{cccccc}
\hline No. & $\theta$ & $i$ & $j$ & $k$ & $\alpha$ \\
\hline 1 & 0.7 & 20 & 20 & 2 & 0.49 \\
2 & 0.9 & 20 & 20 & 1 & 0.89 \\
3 & 20 & 20 & 3 & 0.34 \\
4 & 0.7 & 20 & 20 & 1 & 0.89 \\
5 & 0.9 & 20 & 20 & 1 & 0.59 \\
6 & 0.6 & 20 & 20 & 3 & 0.73 \\
7 & 0.9 & 20 & 20 & 3 & 0.51 \\
8 & 0.8 & 20 & 20 & 2 & 0.81 \\
\hline
\end{tabular}


Analyzing the table it is possible to conclude that a bigger robot's memory coefficient corresponds to a bigger relative receptivity to education (except for line 5).

\section{Conclusions}

According to [6], the psychological parameters described above for robots, are to be assumed as approximate psychological characteristics of humans, therefore the robot's relative receptivity to education can be assumed equal to the human's relative receptivity to education in the first approximation. This can help when modeling humanoid robots as psychological analogs of humans.

Thus, the paper presents mathematical models of characteristics of robots' receptivity to education and the method of approximate calculation of these estimates for a human and a robot by way of example of voice training.

The considered methods of calculation of robot's receptivity to education can be applied for estimation of vocal abilities of deaf and hearing-impaired children; also they can facilitate adaptation of actors to an auditorium where they are supposed to perform.

The presented methods are tested and approved so they can be accepted in the relevant field and applied in a rather short period.

\section{References}

[1] Pensky, O.G. and Chernikov, K.V. (2010) Fundamentals of Mathematical Theory of Emotional Robots. 132p. http://arxiv.org/abs/1011.1841

[2] Pensky, O.G., Sharapov, Y.A. and Chernikov, K.V. (2013) Mathematical Models of Emotional Robots with a NonAbsolute Memory. Intelligent Control and Automation, 4, 115-121.

[3] Pensky, O.G. and Chernikov, K.V. (2013) Mathematical Models of Mental Sets for Robots. In: Isskustvenny Intellect $i$ Prinyatie Reshenii, Russian Academy of Sciences, Moscow, No. 2, 95-99. (in Russian)

[4] ELSYS. (12.12.2012) http://www.elsys.ru/

[5] Chernikov, K.V. (2010) Sound as a Subject for Modelling Emotions of Robots. Investigated in Russia: Electronic Journal. (in Russian) http://zhurnal.ape.relarn.ru/articles/2010/083.pdf

[6] Chernikov, K.V. (2013) Mathematical Models of Robots with a Non-Absolute Memory. PhD Thesis, Perm State University, Perm, 132p. (in Russian)

[7] Chernikov, K.V. (2010) SoundBot-The Program Modelling Mimic Emotional Response of a Robot. Rospatent Certificate of Registration of the Computer Program No. 2010612670. 
Scientific Research Publishing (SCIRP) is one of the largest Open Access journal publishers. It is currently publishing more than 200 open access, online, peer-reviewed journals covering a wide range of academic disciplines. SCIRP serves the worldwide academic communities and contributes to the progress and application of science with its publication.

Other selected journals from SCIRP are listed as below. Submit your manuscript to us via either submit@scirp.org or Online Submission Portal.
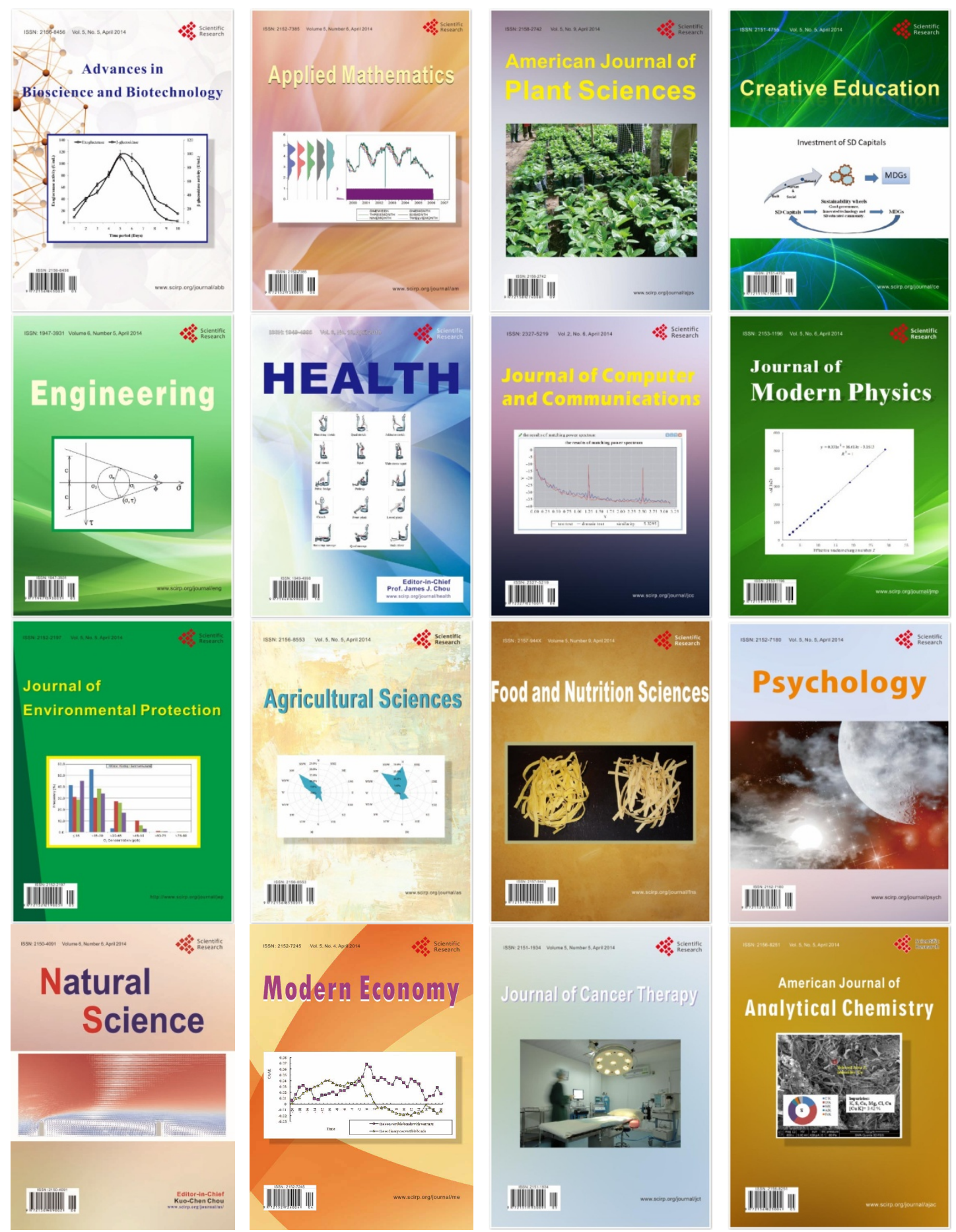